\title{
El rol de las emociones a la hora de resolver el problema del marco ¿Emociones de tipo cognitivas y/o perceptivas?*
}

\author{
The role of emotions in solving the frame problem: \\ Emotions of the cognitive and/or perceptive type?
}

\author{
Por: María Inés Silenzi \\ P.G.I. Análisis de los diversos usos de evidencia empírica \\ para apoyar o atacar posiciones filosóficas \\ Departamento de Humanidades \\ Instituto de Investigaciones Económicas y Sociales del Sur \\ Universidad Nacional del Sur-CONICET \\ Bahía Blanca, Argentina \\ E-mail: misilenzi@gmail.com/misilenzi@uns.edu.ar \\ ORCID: 0000-0002-3003-6261
}

Fecha de recepción: 22 de Mayo de 2018 Fecha de aprobación: 6 de Septiembre de 2018 Doi: 10.17533/udea.ef.n59a04

Resumen. Teniendo en cuenta dos de los principales tipos de emociones, a saber, las emociones perceptivas y las emociones cognitivas, en este trabajo examinaremos cuáles de ellas tiene mayor alcance explicativo a la hora de explicar cómo resolvemos el problema del marco. De otra manera, analizaremos cuáles de las principales características de las emociones del tipo perceptivas y cognitivas resultan adecuadas a la hora de explicar cómo los seres humanos determinamos la relevancia eficientemente. Argumentamos, asumiendo una posición intermedia, que ambos tipos de emociones ofrecen las herramientas necesarias a la hora de explicar cómo los seres humanos resolvemos el problema del marco, debido, por un lado, al carácter experiencial/corporal propio

* El artículo es parte de la producción del Proyecto del Grupo de Investigación denominado Análisis de los diversos usos de evidencia empírica para apoyar o atacar posiciones filosóficas, código: 24/ I261, financiado por la Universidad Nacional del Sur. Una versión resumida fue expuesta como ponencia en el XI Encuentro de Filosofía E Historia de la Ciencia del Cono Sur (AFHIC), que se llevó a cabo en Buenos Aires del 11 al 15 de Junio del 2018. Quiero expresar mi gratitud hacia dos revisores anónimos cuyos valiosos comentarios y sugerencias contribuyeron al mejoramiento de una versión previa del artículo.

Cómo citar este artículo:

MLA: Silenzi, María Inés. "El rol de las emociones a la hora de resolver el problema del marco ¿Emociones de tipo cognitivas y/o perceptivas?". Estudios de Filosofia 59 (2019): 65-95.

APA: Silenzi, M. I. (2019). El rol de las emociones a la hora de resolver el problema del marco ¿Emociones de tipo cognitivas y/o perceptivas? Estudios de Filosofia, 59, 65-95.

Chicago: María Inés Silenzi. "El rol de las emociones a la hora de resolver el problema del marco ¿Emociones de tipo cognitivas y/o perceptivas?”. Estudios de Filosofia n ${ }^{\circ} 59$ (2019): 65-95. 
de las emociones de tipo perceptivas y, por otro lado, a la relación que las emociones de tipo cognitivas establecen con el logro de objetivos frente a una situación determinada.

Palabras claves: emociones, relevancia, problema del marco, emociones perceptivas, emociones cognitivas

Abstract. Considering two of the main types of emotions, namely, perceptual emotions and cognitive emotions, in this paper we will examine which of them has a greater explanatory power for solving the frame problem. Additionally, we will analyze which of the main characteristics of perceptual and cognitive emotions type are appropriate when explaining how human beings determine relevance efficiently. We argue, assuming an intermediate position, that both types of emotions offer the necessary tools to explain how human beings solve the frame problem. This is due, on the one hand, to the experiential/corporal character of the perceptual emotions and, on the other hand, to the relationship that cognitive emotions establish with the achievement of goals when facing a determined situation.

Keywords: emotions, relevance, frame problem, perceptive emotions, cognitive emotions

\section{Introducción}

En los últimos años el estudio de las emociones se ha convertido en un campo de creciente interés para la psicología cognitiva, la filosofía de la mente y las neurociencias, entre otras. Quizás una de las mayores atracciones dentro de este incipiente campo de investigación es la fuerte insatisfacción por la concepción que tradicionalmente han tenido las emociones, lo que desvanece viejos límites entre emoción y cognición. En efecto, las emociones ya no desempeñan un papel pasivo en la racionalidad del sujeto ni se conciben solamente como sensaciones que el sujeto se limita a "padecer" (tales como el dolor, el miedo, la ira) sino que, inversamente, en la actualidad se aprecia que éstas juegan un papel activo y determinante en los procesos cognitivos. En este trabajo nos orientaremos hacia esta última cuestión: el alcance de las emociones para explicar ciertos procesos cognitivos, orientándonos, mucho más particularmente, a aquellos relativos a la relevancia.

Este interrogante, acerca de cómo los seres humanos determinamos relevancia, ha dado origen al problema del marco (frame problem), convirtiéndose en uno de los problemas filosóficos más importantes, y aún vigente, dentro de la investigación cognitiva. A modo introductorio, podemos entender al problema del marco como un problema que cuestiona, de modo general, los procesos cognitivos que implican determinar relevancia, y más particularmente, como el problema que cuestiona cómo un agente, la mayoría de las veces, selecciona información relevante, frente a un vasto conjunto de información, con eficiencia, es decir, adecuadamente y con prontitud. De otra manera, y desde un punto de vista computacional, al tomar 
una decisión frente a una tarea determinada el número de acciones lógicamente posible es virtualmente infinito y el número de estrategias posibles que podrían ser empleadas para llevarla a cabo es mucho mayor, como también resulta infinito el número de consecuencias que de cada una de ellas se deriva. La pregunta que define al problema del marco es: ¿cómo podemos abarcar un espacio virtualmente infinito de posibilidades y determinar relevancia entre tanta cantidad de opciones posibles?

Antes de avanzar, quizás convenga aclarar qué entendemos por "relevancia" o mejor dicho, qué significa, a lo largo de nuestro trabajo, que un agente selecciona "información relevante". Dado que el problema del marco siempre se realiza en el contexto de determinación de una tarea, consideramos adecuada la definición de relevancia que presenta Hjørland (2010, p. 229): "algo (x) es relevante para una tarea $(T)$ si $x$ aumenta la probabilidad de lograr el objetivo $(O)$ que es implicado por $T$ '. Así, y atendiendo a la relación que entre la relevancia y el problema del marco en este trabajo queremos establecer, cierta pieza de información será o no relevante dependiendo de si contribuye, o no, a lograr los objetivos implícitos en la tarea en cuestión. Así, y volviendo a la definición del problema del marco, nuestro problema de interés cuestiona aquellos procesos cognitivos que atienden a los procesos de relevancia, pero también (y a esto se refiere el aspecto eficiente del problema del marco), a la manera exitosa con que la mayoría de los seres humanos lo hacemos. Tal éxito implica, de acuerdo a nuestra definición de relevancia, seleccionar aquella información que nos permita lograr el objetivo encomendado, y eso en un tiempo prudencial.

Si bien éste problema surgió dentro del campo de la inteligencia artificial (McCarthy \& Hayes, 1969), ha sido luego, y aún es, motivo de fuertes y animosos debates dentro del campo de la filosofía de las ciencias cognitivas. Estos debates, de manera muy general, han girado en torno a dos grandes cuestiones: definir en qué consiste realmente el problema del marco (dificultad definicional) y resolverlo definitivamente, si es que se puede (dificultad resolutiva). Ya en otros trabajos (Silenzi, 2014; 2015b) nos hemos dedicado a ambas cuestiones acordando entender el problema del marco, luego de examinar varias interpretaciones, dificultades y aspectos del mismo, como un problema de relevancia (Crockett, 1994; Schneider, 2007; Shanahan, 1997; Pinker, 2005, entre otros). Debido al auge del estudio de

1 Esta definición de relevancia, semejante a la propuesta por Sperber (1986), resultará clave en nuestro examen puesto que está orientada hacia la acción y el logro de objetivos preestablecidos. 
las emociones y la proliferación de éstas sobre distintas cuestiones, nos pareció oportuno proponer un acercamiento filosófico entre este particular problema y el alcance de las emociones a la hora de resolverlo. Por un lado, y de manera general, nuestro recorte obedecerá entonces a los grandes avances que se han producido recientemente con respecto al rol de las emociones, pero también, y de manera particular, a los límites y alcances de las emociones como vía promisoria para resolver el problema del marco.

De manera general, sostenemos que aunque parezca en principio viable la propuesta que apela al rol de las emociones a la hora de resolver el problema del marco, ésta desatiende algunas cuestiones puntuales que, a nuestro entender, deberían examinarse con precisión. Más específicamente, creemos necesario esclarecer: $a$ ) qué concepción acerca de lo que es una emoción implica esta propuesta, $b$ ) qué tipo de emociones contribuirían a resolver el problema del marco $\mathrm{y}$, esto en qué grado $\mathrm{y}$, finalmente, $c$ ) en qué contextos se aplica o no, dicha propuesta (por ejemplo, en la toma de decisiones y/o razonamiento práctico). El esclarecer dichas cuestiones colaboraría en la tarea, no tan sencilla, teniendo en cuenta la poca investigación disponible, de examinar el rol de las emociones como vía promisoria para resolver nuestro problema de interés. Así, y puesto que sería deseable una mayor profundización en la investigación del tema, nuestro objetivo fundamental en este trabajo será orientarnos particularmente a una de estas cuestiones laterales, a saber, ii) qué tipos de emociones contribuirían a resolver el problema del marco.

La riqueza que atesoran las emociones, entre otras razones, se debe a que en ellas convergen gran cantidad de características, disimiles entre sí, lo que complica cualquier examen. Por esta razón, y con el fin de esclarecerlo, nos restringiremos a examinar el alcance, a la hora de explicar el modo en que los seres humanos determinamos relevancia, de únicamente dos tipos de emociones, a saber, perceptivas y cognitivas. Por supuesto, se nos puede objetar que demarcar así esta cuestión, teniendo en cuenta la gran variedad de emociones vigentes, resulta demasiado simplista. Quizás convenga aclarar, que nuestra demarcación se orientará solamente a examinar aquellos tipos de emociones que atraviesan, precisamente, el debate histórico sobre la resolución o no del problema del marco. Puesto que este debate ya ha justificado en otros trabajos (Silenzi, 2014, 2015c) dividir soluciones cognitivistas y postcognitivistas del problema del marco, en esta ocasión atenderemos al alcance de solamente aquel tipo de emociones a las que históricamente algunos filósofos de la mente se han referido a la hora de explicar el modo en lo que los seres humanos determinamos relevancia. 
Dados nuestros objetivos, el artículo se organizará entonces de la siguiente manera. En la sección 2, describiremos resumidamente en qué consiste el problema del marco y, más particularmente, uno de sus principales aspectos, a saber, el aspecto eficiente. En la sección 3, destacaremos las principales características de las emociones de tipo perceptivas y cognitivas y examinaremos algunas propuestas de solución al problema del marco que, respectivamente, las implican. En la sección $\S 4$, pondremos en tensión ambos tipos de emociones y analizaremos cuál de ellas, resulta más adecuada a la hora de resolver nuestro problema de interés. Finalmente, presentaremos nuestras conclusiones en la sección 5. Argumentaremos que ambos tipos de emociones resultan "equipadas" a la hora de explicar cómo los seres humanos resolvemos el problema del marco debido a, por un lado, el carácter experiencial/corporal propio de las emociones del tipo perceptivas y, por otro lado, a la relación que las emociones del tipo cognitivas establecen con el logro de objetivos frente a una situación determinada.

\section{El aspecto eficiente del problema del marco}

Con respecto a la dificultad definicional del problema del marco, ya en otros trabajos hemos dilucidado varios aspectos del problema, a saber, el aspecto lógico, eficiente, epistemológico y explicativo (Silenzi, 2015a). Puesto que la mayoría de las soluciones y tratamientos del problema del marco afrontan el desafío de resolver principalmente a uno de estos aspectos, a saber, el aspecto eficiente del problema del marco, en este trabajo nos detendremos en este particular aspecto considerado el "fenómeno fundamental del problema del marco". De manera general, éste se refiere a la manera en que un agente selecciona la información relevante entre un vasto conjunto de información, de manera tal que logra realizar la tarea eficientemente. Así hemos denominado a este aspecto "eficiente" para acentuar la adecuación, prontitud y éxito con que la mayoría de las veces los humanos determinamos relevancia, o, con otras palabras, para enfatizar la eficiencia con la que los seres humanos determinamos relevancia. En la literatura al respecto suele aceptarse que a las personas parece "venirse a la mente" solamente la información relevante y eso en el momento preciso y de manera adecuada; pues bien, este fenómeno da cuenta primordialmente del aspecto eficiente. Véase que así descrito, este aspecto refleja cierta regularidad exhibida por los seres humanos a la hora de determinar relevancia, dando por supuesto que las personas suelen determinar qué es relevante para una tarea apropiadamente y con prontitud, aunque, por supuesto, de vez en cuando, 
surjan errores. Uno de los ejemplos claves del aspecto que nos interesa es el que presenta Daniel Dennett en su memorable ensayo publicado en 1984. Este ejemplo ha sido descrito en numerosos trabajos a la hora de introducir el problema del marco (Silenzi, 2015b), puesto que es clave en la comprensión de las divergencias entre el modo en que los seres humanos resolvemos el problema del marco y la (supuesta) imposibilidad de ser resuelto por agentes artificiales. Más particularmente, el autor introduce distintas situaciones en la que un agente artificial no logra llevar a cabo una tarea, desactivar una bomba, debido a su (supuesta) incapacidad por determinar la relevancia eficientemente. Con otras palabras, el autor describe varias situaciones que manifiestan cómo un robot, frente a una tarea determinada, no puede decidir qué hacer o cómo actuar, pues no tiene la capacidad suficiente para seleccionar, con prontitud de entre todas las posibilidades (acciones) existentes, qué acción resulta más adecuada o relevante para lograr llevar a cabo exitosamente su tarea.

Veamos particularmente un ejemplo que ilustra claramente cómo se vinculan el problema del marco con aquellos procesos cognitivos que implican relevancia. Para entender a este problema, Dennett apela a un experimento mental en el que invita a imaginarnos un robot imaginario $R l$ al que sus diseñadores le fijan la tarea de recuperar su batería de repuesto. La batería se encuentra dentro de un carro en una habitación cerrada en la que hay una bomba programada para estallar en poco tiempo. $R 1$ hace la hipótesis de que una cierta acción Sacar (Carro, Habitación) le permitiría cumplir su tarea. Entra pues a la habitación y saca el carro con su batería de repuesto. Desafortunadamente, al estar la bomba también dentro del carro, el robot estalla a los pocos segundos.

Los diseñadores de $R 1$, para evitar tal explosión, creen que es necesario que el robot no sólo considere las implicaciones intencionadas de sus actos, sino también las implicaciones secundarias. Así, diseñan el siguiente robot, llamado $R 1 D 1$, que también encuentra la batería y diseña un plan de acción; sin embargo, cuando acaba de deducir que quitar el carro hacia afuera de la habitación no haría cambiar el color de las paredes de ésta y está embarcándose en la comprobación de la siguiente implicación, la bomba explota.

Con esta situación, los diseñadores consideran necesario enseñarle al robot a diferenciar, a través de mecanismos de inferencia, aquellas implicaciones que son pertinentes y que deben tenerse en cuenta al diseñar un plan de acción, de aquellas otras que no lo son. El diferenciar tales implicaciones permitiría que el robot no se quede congelado considerando todas y cada una de las implicaciones, relevantes 
e irrelevantes. Llaman a este tercer robot $R 2 D 1$ y le dan la misma orden. Cuando el robot localiza la batería, se sienta afuera de la habitación. Los diseñadores, angustiados por ver que el robot se queda congelado de nuevo, le gritan que haga algo y el robot responde: “ ¡Lo estoy haciendo (...) estoy ignorando diligentemente las miles de implicaciones que determiné improcedentes! ¡Tan pronto descubra una implicación improcedente, la sumo a la lista de las que debo ignorar!" Así, mientras el robot se concentra en omitir consideraciones innecesarias, explota la bomba y el robot se queda sin batería. A través de este ejemplo, es claro que un requisito necesario para poder solucionar el problema del marco es lograr que el programa construido permita tomar las decisiones adecuadas, y activar en consecuencia, dentro de un tiempo razonable.

Ahora bien, ninguno de los tres robots ha solucionado, según Dennett, el problema del marco. El primer robot no tiene un mecanismo que le permitiera considerar de manera completa las implicaciones de sus actos. El segundo robot tiene esta capacidad pero carece de la capacidad de distinguir las consecuencias relevantes de las irrelevantes. Y finalmente, el tercero posee esta capacidad pero carece de la habilidad de coordinar esa distinción con las acciones necesarias para la tarea, al menos, en un tiempo prudencial. Así, se puede argumentar que una de las preguntas que Dennett tiene en mente sobre el problema del marco es la siguiente: ¿cómo es que al momento de realizar una determinada tarea, el sistema cognitivo es capaz de determinar relevancia y generar, expeditivamente una secuencia de acciones, sin terminar paralizado computacionalmente? En otras palabras, Dennett dejaría entrever que una solución al problema del marco debe dar cuenta de ciertas habilidades de la cognición humana al realizar una tarea dada como, por ejemplo, comprender el alcance de los efectos secundarios de las propias acciones en tiempo real, discriminar las implicaciones relevantes de las inferencias y relacionar estas implicaciones con la interacción con el ambiente.

Veamos otro caso. El ejemplo de Lowe (2001, p. 192) invita a explicitar cómo es que los seres humanos, a pesar de las limitaciones a las que irremediablemente debemos enfrentarnos, logramos determinar la relevancia exitosamente la mayoría de las veces. Pensemos que estamos eligiendo un menú en un restaurante y que de golpe suena la alarma pudiéndose observar, desde nuestro asiento, la ventana ubicada justo enfrente de nosotros desde donde sale el humo: en esta situación debemos elegir entre cruzar y hacer algo con respecto al incendio, o seguir eligiendo el menú: ¿cómo se comportaría un agente artificial 
en estas circunstancias? Pues bien, ni el programa de petición de menú ni el de ayuda en caso de incendio pueden especificar por sí mismos qué hacer, pues cada uno de estos programas está dedicado a su propio terreno de competencia. Incluso con un diseño de tipo transversal, aparece una infinidad de combinaciones posibles. Lo que en realidad se dificulta en ambos ejemplos es modelar nuestra capacidad de determinar la relevancia, la cual nos permite pasar continuamente de una actividad inteligente a otra frente a la aparición de nueva información y establecer relevancia eficientemente frente a nuevas prioridades. En otras palabras, lo que ambos agentes no logran superar es la denominada dificultad de la vastedad de información. Esta dificultad puede verse claramente si se considera la siguiente estrategia ingenua para resolver el problema del marco: analizar cada una de las piezas de información del sistema y evaluar, en cada caso, si es o no relevante para realizar la tarea en cuestión. Claramente, esta estrategia no es factible de aplicar cuando la cantidad de información que posee un agente es abrumadoramente vasta, como es en el caso de los seres humanos. Pero, y esto será clave para nuestro análisis, tampoco lo es debido a la temporalidad que apremia al agente: desactivar una bomba o reaccionar frente a un incendio implica, para lograr llevar a cabo la tarea exitosamente, determinar la relevancia dentro de un tiempo prudencial. El "acoplarse eficientemente" a distintas situaciones resulta primordial para no quedar paralizado frente a los objetivos propuestos y la tarea encomendada. Resulta fundamental que, en el plano de la toma de decisiones, el agente, como resultado de su "ajuste" a la situación en la que se encuentre inmerso, "actúe" sin quedar detenido. Teniendo en cuenta la definición de relevancia propuesta, parecería, al menos en primera instancia, que al momento de determinar la relevancia, la adaptación al entorno es lo que permite al agente "actuar" sin quedar inmovilizado. En síntesis, la flexibilidad y adaptación con el entorno es lo que permite a los seres humanos resolver el aspecto eficiente del problema del marco e incluso, y en lo que concierne a la posibilidad de adaptación, es lo que permite explicitar por qué, la mayoría de las veces, lo hacemos con cierto éxito. Así, sostenemos que es imprescindible, a la hora de explicar el aspecto eficiente del problema del marco, tener en cuenta al agente anclado (situado) en un mundo real que "pisa sobre el mundo" (a esto nos referiremos cuando, oportunamente, destaquemos el carácter experiencial/corporal de las emociones perceptivas) y que "accione" sin quedarse paralizado, teniendo en cuenta los objetivos y la tarea encomendada (a esto nos referiremos cuando, oportunamente, destaquemos el rol activo de la emociones cognitivas). 
Introduciéndonos ya en el plano de las emociones, nos parece conveniente aclarar antes de continuar con nuestro examen, qué entendemos por la noción de emoción. Puesto que ésta resulta clave en nuestro trabajo se nos puede objetar la ausencia de una definición clara de lo que entendemos por ella. Como ocurre con la definición del problema del marco, ofrecer una definición rigurosa de lo que "es" una emoción no es una tarea sencilla. Por eso preferimos describir aquellos aspectos de las emociones que resultan claves para nuestro análisis, quedando pendiente en nuestra agenda de investigación profundizar qué concepción de emoción es la más adecuada a la hora de explicar el problema del marco. Por ahora, y a modo de estrategia, destacaremos, en referencia a algunos de sus rasgos más salientes, la distinción entre disposiciones y experiencias emocionales, puesto que resultarán claves para nuestro análisis. Las disposiciones emocionales se refieren a aquellos fenómenos en los que el sujeto posee cierta predisposición a comportarse de una determinada manera frente a una situación determinada, o con algunas personas en especial. Por ejemplo, la predisposición hacia un hijo es la que dispone a cuidarlo en caso de que esté enfermo o a cocinarle cuando tenga hambre, entre otras tantas cosas que hacemos por los hijos. Pero el hecho de que a veces estemos preocupados por ellos no implica que los padres se encuentren preocupados y pendientes todo el tiempo de sus hijos, y eso último no significa, por supuesto, que no se los ame. Esta disposición se diferencia de la experiencia emocional, en donde sí se experimenta, "se sienten", las vivencias implicadas en ciertos actos tales como las que se sienten al amamantarlos y dormirlos en brazos cuando son pequeños o, al abrazarlos y besarlos. Esta última manera de entender las emociones, a la que atenderemos particularmente en este trabajo, implica la experiencia, la dimensión corporal, el cuerpo, o de otra manera, el sentir con el cuerpo. Pero también implica, tal como lo ilustran nuestros ejemplos, cierta duración temporal en la que el sujeto se enfrenta y actúa frente a una situación (Schwitzgebel, 2010). Esta manera de interpretar la emoción remite a lo que el sujeto siente en esa, y no otra, situación determinada y a la acción que esa tarea, y no otra, conlleva. Volviendo al aspecto eficiente del problema del marco, véase que esta manera de entender la emoción acentúa algunos de sus rasgos fundamentales: a saber, la "situacionalidad" y la acción. Puesto que la acción y el factor situacional/temporal son claves a la hora de explicar el problema del marco, y especialmente su aspecto eficiente, entendemos que el carácter experiencial/corporal y el rol activo de las emociones recién descritos resultan compatibles y acordes a nuestros propósitos, aunque, por supuesto, esto no excluye que otros rasgos y maneras de interpretar las emociones también lo sean. Aclarada esta cuestión, es hora de distinguir, suponiendo que tal distinción sea 
adecuada, dos de las principales teorías acerca de la emociones, a fin de estimar, al momento de abordar teóricamente al problema del marco, solamente dos tipos de emociones.

\section{Teorías perceptivas y cognitivistas}

Como todo campo de investigación incipiente, las emociones han despertado el interés de varios autores proponiéndose varias y distintas teorías sobre lo emocional. Estas teorías resultan, por su particularidad, difíciles de agrupar dentro de un mismo marco teórico haciendo imposible postular una visión global acerca de las distintas teorías de emociones sin caer en ciertos reduccionismos. Creemos que, para fines expositivos y metodológicos, la división entre teorías perceptivas y cognitivistas resulta suficiente para nuestros propósitos, aunque, por supuesto, se nos puede objetar que cualquier subsunción de las diversas teorías actuales sobre la emoción en sólo dos grupos de teorías implique un cierto riesgo de simplificación y reduccionismo. Cabe aclarar que la manera en que las hemos agrupado resulta como consecuencia de enfatizar exclusivamente las versiones más sencillas de cada grupo con el propósito de destacar sus diferencias más fundamentales. De nuevo, podemos encontrar ciertos matices entre ambos grupos e inclusive dentro de cada grupo por separado. Por supuesto, atender a estos matices resulta fundamental a la hora de postular cierta cartografía acerca de las emociones, siendo nuestro objetivo en esta sección solamente postular sus rasgos más distintivos.

Esta división, apropiada para nuestros propósitos, obedece, en primer lugar, a dos criterios clasificatorios: sensación e intencionalidad. Ambos criterios nos permiten abarcar el grueso del estado de la cuestión sobre la investigación acerca de la teoría de las emociones y delimitar nuestro trabajo. Así, de manera muy general, las teorías cognitivistas conciben las emociones como estados cognitivos y las teorías perceptivas como fenómenos corporales. Por supuesto, esto no excluye la existencia de teorías perceptivas que hagan hincapié en el carácter de intencionalidad de las emociones, y esto en distintos grados. El intento de estas teorías por derivar la intencionalidad de las emociones, como el de las teorías cognitivistas por abarcar el cuerpo, y no considerar las emociones exclusivamente como juicios abre un abanico de propuestas que, estimarlas en su totalidad a la hora de resolver el problema del marco excedería el espacio disponible. 
En segundo lugar, otro criterio que colabora en tal división obedece al estado de los elementos de las emociones. Denominamos teorías cognitivistas a aquel grupo de teorías que entienden las emociones como estados cognitivos (sean creencias, pensamientos, juicios, deseos, etc.), siendo la estrategia utilizada para denominarlas de esa manera, y a modo de reacción teórica dentro de la literatura del área, el carácter activo de estos elementos. De esta manera, denominamos teorías cognitivistas de las emociones a aquellas teorías que destacan como elementos activos de las emociones los estados cognitivos. En contraposición, las teorías perceptivas de las emociones destacan como elementos activos de las emociones las sensaciones corporales. Así, y teniendo como referencia el campo de la toma de decisiones, puesto que es allí donde ubicamos al problema del marco, e introduciendo ya sus principales diferencias, las teorías cognitivistas enfatizan los aspectos cognitivos de las emociones concibiéndolas como juicios (creencias, pensamientos, deseos, etc.) sobre el mundo, mientras que el segundo grupo enfatiza los aspectos cualitativos de las emociones interpretándolas a través del modo fenoménico en el que son sentidas corporalmente, es decir, haciendo hincapié en la relación de las emociones como reacciones corporales.

Veamos el siguiente ejemplo que nos ilustrará mucho más elegantemente las diferencias descritas. Supongamos que estamos andando en bicicleta y que un auto a gran velocidad pasa a nuestro lado, por poco rozándonos, y que casi nos atropella, obligándonos a frenar de golpe. Entre las emociones que nos puede invadir en esta situación particular sentiremos, en primer lugar, miedo, y luego, enojo. De acuerdo a las teorías perceptivas, y desde un punto de vista fisiológico, el miedo y el enojo se vinculan con reacciones fisiológicas y no con juicios sobre esa situación particular. Seguramente sentiremos, al momento en que el conductor pasa a toda velocidad a nuestro lado, ciertos cambios corporales: se nos acelerará el pulso y la presión, transpiraremos, etc. De acuerdo a este grupo de teorías, las emociones son respuestas cuasi reflejas ante determinados estímulos, relacionadas con los cambios corporales que experimentamos (Thagard, 2008, p. 248). Entre los mayores representantes de estas teorías se encuentran y Damasio (2001), James (1985 y 1989), Lange (1885) y Prinz (2004; 2006) entre otros. Por otro lado, y en contraste con lo anterior, de acuerdo a las teorías cognitivistas el miedo se deriva de la inferencia de que corremos cierto riesgo físico, que amenaza nuestra salud y supervivencia, mientras que el enojo resulta como consecuencia del juicio según el cual el conductor nos puso en 
peligro. Así, este grupo de teorías pone el acento en los juicios cognitivos y en las emociones como formas de valoración. Entre los mayores representantes de esta postura se encuentran Hansberg (1996), Nussbaum (2008), Oatley (1992) y Solomon (1993), entre otros.

Volviendo a las teorías perceptivas, las emociones no son las causas de los cambios corporales que percibimos en una situación determinada, sino que la percepción de tales cambios corporales son las emociones mismas. Con otras palabras, aquello que causa la emoción no es parte constituyente de la emoción misma, sino que la emoción es la percepción del efecto en los cambios fisiológicos del agente. En este sentido, la tesis principal de estas teorías es que una emoción es un tipo de percepción. Como ejemplos clásicos de estas sensaciones se suele citar la aceleración del corazón, el rubor del rostro, la sudoración de las manos, etc. Estas emociones, y esto resultará clave para nuestro análisis, son de carácter experiencial/corporal. Así, la percepción de mi cuerpo temblando, provoca el miedo y la de mi corazón latiendo apresuradamente provoca el enojo.

Las teorías cognitivistas destacan primordialmente el componente cognitivo de las emociones y, en su versión más radical, consideran que éste es el único componente que constituye la emoción, al punto tal de ser idéntica a un juicio o pensamiento. Por ejemplo, en el caso del enojo, la emoción es "enjuiciar" el mundo como ofensivo y como peligroso en el caso del miedo. Estas teorías identifican las emociones con juicios, ideas, pensamientos a través de los cuáles conocemos rasgos emocionales "del mundo". En el ejemplo anteriormente citado, la situación particular en la que un auto casi nos atropella, se presenta como "peligrosa", lo que supone una estimación o valoración de esa situación. Así, debido a la intencionalidad de este tipo de emociones, la cual puede entenderse como el carácter de las emociones de dirigirse hacia el mundo y poder referirse así hacia objetos, situaciones o eventos del mundo externas al agente, el sujeto "valora" el mundo: en este ejemplo, conocemos lo peligroso de la situación a través del miedo. Pero, además, al identificarse las emociones con estados cognitivos, estas teorías las hace participes de la racionalidad de los sujetos al momento de tomar decisiones. Por supuesto, podemos encontrar varios matices entre ambos grupos de teorías como consecuencia del empeño por parte del grupo de las teorías cognitivistas por abarcar en su análisis el cuerpo y, del grupo de las perceptivas por derivar la intencionalidad de las emociones. Sin embargo, y como ya lo hemos destacado, hemos caracterizado los rasgos más sobresalientes de cada tipo de teorías. 
Puesto que ninguna de estas opciones resulta excluyente, nos pareció conveniente orientarnos en esta sección, y de modo introductorio, hacia las teorías más significativas acerca de las emociones para referirnos luego, mucho más extensamente, a las emociones de tipo perceptivas y cognitivas, pues nuestro objetivo primordial es estimar el tipo de emociones que históricamente se han tenido en cuenta a la hora de explicar el modo en que los seres humanos determinamos relevancia. En este sentido, y a modo de estrategia metodológica para poder llevar a cabo nuestro objetivo fundamental, en nuestro trabajo nos hemos referido, en primer lugar, a las principales teorías de las emociones para luego abocarnos hacia la posibilidad de que sólo dos tipos de emociones resuelvan el problema del marco. Así, hemos querido clarificar esta cuestión para evitar posibles confusiones. Descritas de manera muy general dos de las teorías más importantes acerca de las emociones, nos referiremos de ahora en más solamente a dos tipos de emociones: cognitivas y perceptivas. Quizás convenga aquí aclarar que, con respecto al reconocimiento de las emociones, tradicionalmente se ha aceptado una distinción entre, por un lado, las emociones básicas (primarias) que serían emociones de tipo perceptivas, y, por otro lado, emociones derivadas (combinaciones de emociones básicas más creencias y estados doxásticos) que serían emociones de tipo cognitivas (puesto que implican procesos de evaluación cognitiva). Las primeras, se definen como reacciones emocionales de carácter universal e innato, y abarcan alegría, tristeza, asco, sorpresa, miedo y enojo y las segundas no poseen una expresión facial o corporal claramente identificable dado que se encuentran asociadas a variaciones culturales y al aprendizaje, y abarcan culpa, orgullo, celos, desprecio y vergüenza entre otras (Ekman, Sorenson \& Friesen 1969; Ekman \& Friesen 1971; Ekman, 1993 \& Plutchik, 1980). Así, puede observarse qué emociones particulares forman parte de los tipos (conjuntos) de emociones a los que de ahora en más nos referiremos. Aunque no atenderemos a cada una de las emociones individuales (alegría, enojo, etc.) queda pendiente en nuestra agenda de investigación examinar todas y cada una de ellas en particular. A continuación entonces, revisaremos algunas propuestas de solución al problema del marco que apelan a dos grandes tipos de emociones, perceptivas y cognitivas, siendo nuestra estrategia, con base a la poca literatura disponible, sistematizar distintas propuestas de acuerdo al tipo de emoción a la que, como argumentaremos, cada una de ellas apuntan. 


\section{1. Problema del marco, racionalidad y emociones de tipo cognitivas}

Entre los aportes que ofrece la investigación sobre las emociones, algunos filósofos de la mente destacan aquellos que colaboran en la comprensión de la racionalidad. Con respecto al campo de la toma de decisiones, las nociones más asociadas con la racionalidad son la coherencia y consistencia y, con respecto a la esfera de la acción, la maximización de la utilidad esperada. Para De Sousa (1980), estas nociones son totalmente criticables pues, por sí mismas, son incapaces de guiar a un agente, natural o artificial, hacia cualquier curso de acción particular. Como ya hemos descripto, el problema del marco refleja aquella situación en la que, frente a una tarea determinada, los seres humanos determinamos relevancia eficientemente, es decir con adecuación y prontitud, logrando así llevar a cabo exitosamente su tarea. De otra manera, y para De Sousa, desde un punto de vista computacional, al tomar una decisión el número de acciones lógicamente posible es virtualmente infinito y el número de estrategias posibles que podrían ser empleadas para llevarla a cabo es mucho mayor, como así también resulta infinito el número de consecuencias que de cada una de ellas se deriva.

Visto de esta manera, la evaluación de alternativas posibles a la hora de llevar a cabo una tarea podría no finalizar nunca, terminando el agente paralizado sin lograr tomar una decisión. Pues bien, para este autor, y de acuerdo al modelo estándar de elección racional, el problema del marco refleja esta situación particular en la que, resumidamente, un agente se enfrenta a una explosión combinatoria de posibilidades de elección.

Ahora bien, es notable que para De Sousa, el problema del marco así descrito afecta solamente a agentes artificiales, convirtiéndose en uno de los mayores obstáculos dentro del campo de la inteligencia artificial, pero no a los seres humanos puesto que nosotros, a diferencia de agentes artificiales, poseemos emociones. Las emociones, frente a la situación descrita, contribuyen en la solución del problema del marco pues funcionan como fuentes de relevancia entre objetos de atención, líneas de investigación y estrategias inferenciales (De Sousa, 1996, p. 137). Véase que directamente De Sousa relaciona las emociones con aquellos procesos cognitivos que implican relevancia, procesos a los que, como ya hemos descripto (recuérdese el ejemplo de Dennett), se orienta el problema del marco. Quizás resulte conveniente aquí aclarar que en la literatura sobre el tema podemos 
observar que, de manera general, algunos de los debates acerca de la relevancia epistemológica de las emociones al explicar algunos de los principales problemas de las ciencias cognitivas, giran en torno a las varias y distintas funciones epistémicas que éstas prometen, a saber, fuerza motivacional, eficiencia, acceso a los hechos y creencias, contribución no-proposicional al conocimiento y, entre otras, saliencia y relevancia (Dohrn, 2008). De entre estas funciones, algunos autores como De Sousa, y como luego veremos Damasio, enfatizan la función epistémica de la relevancia, vinculando, así, y como también hacemos nosotros, el problema del marco con la relevancia.

Antes de avanzar, es necesario dilucidar que, para que la relación de De Sousa con el problema del marco pueda establecerse, es menester que las emociones a las que haga referencia sean solamente de tipo cognitivas. De otra manera, su propuesta teórica, que propone vincular el rol de las emociones con el problema del marco, solamente se puede sostener si las emociones a las que se refiere son intencionales y ocupan un rol activo en nuestros procesos racionales, es decir, si son cognitivas. Es la exclusividad de esta relación la que aquí queremos apuntar. Como podrá observarse a continuación, De Sousa declara explícitamente que las emociones que tiene en mente son de tipo cognitivas. Lo que nosotros acentuamos es que además, y lo que a nuestro entender el autor no explicita, es condición que así sean para establecer la relación con el problema del marco tal como él lo interpreta y de acuerdo a su idea de racionalidad. Como veremos luego (sección 4), a propósito de la posibilidad de combinarse emociones cognitivas y perceptivas al momento de determinar relevancia, De Sousa excluye esta posibilidad pues solamente es posible que las emociones cognitivas sean parte de este proceso: únicamente puede vincularse, para este autor, el rol de las emociones con el problema del marco si éstas son de tipo cognitivas, de lo contrario, no podrían relacionarse. Veamos más detenidamente esta relación atendiendo a los elementos de las emociones cognitivas.

La concepción de las emociones que defiende De Sousa sostiene que éstas son juicios de tipo valorativos y que son de naturaleza personal, es decir, que provienen del mismo sujeto. Así, para este autor, las emociones son juicios valorativos de objetos y situaciones que son sólo importantes para el sujeto. Esta manera de entender las emociones permite que el sujeto "enjuicie" el mundo en el que se encuentra inmerso en relación a sus propios intereses, creencias, etc. Esto, a su vez, le permite reflexionar, valorar y realizar inferencias sobre él. Ahora bien, definir a una emoción como un estado cognitivo valorativo refleja el principal intento de 
De Sousa por racionalizar las emociones, y atribuirle al sujeto un papel activo en su control. En este sentido, solamente las emociones que enfatizan tales estados cognitivos, es decir, y de acuerdo a nuestra clasificación, solamente las emociones cognitivas, le resultan racionales; a la inversa, las emociones que no dicen nada acerca de lo que piensa, infiere y estima un sujeto, es decir, que no enfatizan estados cognitivos, le resultan irracionales.

Precisamente, y a nuestro entender, el mayor poder explicativo de su propuesta, recae en la posibilidad de realizar inferencias "racionalmente", posibilidad que está vinculada solamente a las emociones cognitivas, según nuestro análisis. Para este autor, la principal relevancia epistemológica de las emociones gira en torno, entre las varias y distintas funciones epistémicas que éstas prometen, a la función epistémica de la saliencia y relevancia de las emociones la cual, nuevamente, implica realizar juicos valorativos que provengan desde el sujeto hacia el mundo y que le permitan inferir, reflexionar, etc. "racionalmente" y de acuerdo a sus creencias, intereses, pensamientos. Puesto que, como hemos visto, solamente le atribuye racionalidad a las emociones que enfatizan tales estados cognitivos, entendemos que la función de saliencia y relevancia de las emociones, defendida por el autor, es llevada a cabo solamente por emociones de tipo cognitivas.

El cuestionamiento que delimita el problema del marco fundamenta aún más nuestro razonamiento. La pregunta que define al problema del marco para De Sousa es: ¿cómo podemos abarcar un espacio virtualmente infinito de posibilidades y determinar relevancia entre tanta cantidad de opciones posibles? Pues bien, es el tipo de emociones cognitivas el que, solamente y desde un punto de vista teórico, resolvería esta cuestión, al "recortar información", garantizando así la tratabilidad computacional del problema del marco, o en otras palabras, el único tipo de emoción que resolvería la dificultad de la vastedad de información. Los seres humanos, al poseer emociones, efectuamos sin mayores complicaciones el acotamiento necesario, determinando aquellas acciones y consecuencias "realmente relevantes" sin malgastar tiempo en deducciones irrelevantes. Véase el acento en los juicios cognitivos y en las emociones como formas de valoración: el autor le aplica a las emociones, las categorías de justificación, adecuación o racionalidad, al estimarlas como "verdaderamente relevantes", categorías aplicables solamente a las emociones de tipo cognitivas. Pero también, puede reconocerse la referencia al tipo de emociones cognitivas al establecer, de manera primordial, la relación de las emociones con el logro de objetivos. En efecto, al examinar el rol de las emociones a la hora de resolver el problema del marco, el autor postula una situación de 
toma de decisiones con diversos objetivos en conflicto y un entorno muy variable, donde las emociones (de tipo cognitivas) ofrecerían una valoración muy resumida de la situación para actuar, frente a la tarea y a los objetivos preestablecidos (sin quedarnos enredados en inferencias innecesarias). Recordemos que, para poder resolver eficientemente el problema del marco, y de acuerdo a lo que entendemos por relevancia, es menester que las emociones nos motiven a "actuar" en virtud de los objetivos propuestos, siendo capaces de ser maleables y versátiles en el dominio de respuestas posibles frente a situaciones cotidianas e inesperadas que puedan darse. Pues bien, son precisamente las emociones de tipo cognitivas las que permiten aplicar nuestro conocimiento de modo adecuado a las circunstancias pertinentes y actuar, o en otras palabras, las que nos permiten determinar relevancia eficientemente.

En síntesis, argumentamos que el tipo de emociones a las que se refiere De Sousa son de tipo cognitivas debido, entre otras razones, a: $i$ ) el carácter inherentemente racional de nuestras emociones (el cual nos permite inferir, evaluar, estimar, etc. "racionalmente" información), ii) el acento en los juicios cognitivos y en las emociones como formas de valoración (intencionalidad) y iii) la relación de las emociones con el logro de objetivos (rol activo). Por supuesto, esto no excluye la referencia en su propuesta a otros tipos de emociones; solamente destacamos, a nuestro juicio, el tipo de emoción sobresaliente.

Aunque nos hemos detenido en la propuesta de De Sousa, debido a su trascendencia, no queremos dejar pasar algunas soluciones similares. Para Elgin (1996) algunos aspectos de una situación demandan automáticamente nuestra atención eclipsando otros factores epistémicamente accesibles, siendo las emociones las que "nos inclinan" hacia algunas facetas particulares de esa situación particular. Para este autor, si las emociones acentúan un factor como saliente, o al menos se presenta como siéndolo, entonces presuntivamente éste "resulta significativo o relevante". Así Elgin (2008), como De Sousa, también ha defendido la relevancia epistemológica de las emociones y otros estados afectivos por la vía de mostrar que estos estados cumplen funciones propiamente epistémicas, indispensables no sólo en los procesos de construcción del conocimiento sino también en los procesos de evaluación. En este sentido, entiende, al igual que De Sousa que, en los procesos de determinación de relevancia (lo cual implica evaluar, deliberar, reflexionar, etc.), las emociones cognitivas, entendidas éstas como juicios acerca del mundo, juegan un rol esencial. Así, y como también lo plantea De Sousa, éstas son una "faceta de la razón" que proporcionan recursos que sirven a fines epistémicos. Elgin 
particularmente estima que si, a la inversa, las emociones no fueran cognitivas, la información que ofrecerían sería "ligeramente torcida", no porque las emociones no fueran genuinas, sino en el sentido de que no se relacionarían con la situación en la que en el sujeto se encuentra tal como éste quisiera relacionarse o con los objetivos que persigue. Para ilustrar esto, considere la situación cognitiva de un sujeto que buscando información sobre Florida, Buenos Aires, e inadvertidamente busca información sobre Florida, Estados Unidos. Aún si esta información puede ser interesante, ésta no es la que el sujeto desea o necesita saber. Si una emoción está bien fundada, es decir si es cognitiva y racional, ésta se "sintoniza" adecuadamente al sujeto con situación. Así, los factores que destacan las emociones de tipo cognitivas son relevantes y significativos, resultando ser una fuente de información que, en tales circunstancias, vale la pena tener.

Véase que la fiabilidad "emocional racional" que adscribe Elgin (2008) a las emociones da cuenta, nuevamente, de $i$ ) el carácter inherentemente racional de nuestras emociones (el cual nos permite inferir, evaluar, estimar, etc. información racional, bien fundada y adecuada a nuestros propósitos). Para este autor, los seres humanos "somos presos de la sobrecarga de información masiva" (Elgin, 2008, p. 43) y es un hecho que conocer, comprender, percibir o discernir algo requiere pasar por alto mucha información, pero la pregunta que considera fundamental es: ¿qué se debe pasar por alto? o, de otra manera, ¿qué es lo irrelevante?, y aún más ¿cómo lo determinamos y sabemos que lo hacemos adecuadamente? (Brun et al., 2008, p. 43). Pues bien, las emociones colaborarían en la tarea de determinar relevancia apropiadamente no sólo ii) al ofrecer una valoración muy resumida de la situación, es decir, al estimar los aspectos realmente relevantes de una situación (concéntranos sólo en éstos y sin que nuestros limitados recursos cognitivos, y el poco tiempo disponible, se ocupe de cuestiones irrelevantes) sino que también iii) nos orientarían a la acción frente a una tarea determinada. Claramente, y de acuerdo al rol descrito, el tipo de emociones al que se refiere Elgin en su propuesta es de tipo cognitiva.

\subsection{Problema del marco, marcadores somáticos, y emociones perceptivas}

Sin dudas, Damasio $(2001 ; 2010)$ es uno de los filósofos de la mente que más ha tratado el problema del marco. A él se le atribuye explicar a las emociones a través de su teoría del marcador somático, entendiendo a las emociones como 
marcadores somáticos, concibiéndolas como aquellas señales, relacionadas con factores fisiológicos, enviadas desde el cerebro. Además, su propuesta defiende explícitamente el papel de las emociones perceptivas al momento de determinar relevancia, lo que justifica, en primera instancia, la elección de la teoría de Damasio entre otras. En su tratamiento, y volviendo a las funciones epistémicas de las emociones a la hora de explicar nuestros procesos cognitivos, Damasio acentúa, tal como lo hacemos nosotros, la función de saliencia de las emociones y con ello, al rol de las emociones a la hora de abordar el problema del marco. Esta relación, entre las emociones y los procesos cognitivos que implican relevancia, no sólo coincide con la relación que en este trabajo hemos planteado, sino que además coincide con la de De Sousa. De esta manera, estimamos en este trabajo y de manera coherente, el alcance del rol de las emociones al abordar el problema del marco, teniendo en mente una misma interpretación del problema del marco, a saber, sobre el problema del marco entendido como un problema acerca de la relevancia. ${ }^{2} \mathrm{Si}$ estimamos el rol de las emociones (o cualquier otra solución) sobre interpretaciones distintas del mismo problema, complejizaríamos el intento de explicarlo. Si atendemos al tratamiento histórico del problema del marco (Kamermans \& Schmits, 2004), podemos observar que se han ofrecido soluciones de tipo lógicas a interpretaciones de tipo filosóficas, y a la inversa, soluciones filosóficas a interpretaciones de tinte lógicas del mismo problema, generándose varios debates y controversias producto del no esclarecimiento de la particular relación antes mencionada. Por esta primera razón, nos pareció apropiado examinar una propuesta acerca del rol de las emociones que coincida con la interpretación del problema del marco propuesta y que evite tales confusiones. Asimismo, al explorar la literatura sobre el tema podemos observar que el tratamiento del problema del marco por parte de investigadores cognitivos acerca de la emoción es casi nulo y que, la mayoría de las veces, lo hacen de manera introductoria. Por esta segunda razón, nos parece destacable predominar, el tratamiento profundo que del problema hace Damasio por sobre otros, que o no lo hacen o sólo lo hacen sin mucho detalle. Finalmente, el hecho de que algunos estudios científicos apoyan la propuesta de

2 Estas afirmaciones coinciden con la relación, ya mencionada y profundizada en otros trabajos (Silenzi, 2014; 2015b) entre la dificultad definicional (la dificultad por definir de qué trata el problema del marco y resolutiva (cómo resolverlo) del problema del marco: antes de estimar cualquiera de las dos soluciones del problema del marco es menester atender a una misma interpretación del problema del marco. En este caso, la propuesta acerca del rol de las emociones a la hora de abordar, desde un punto de vista teórico, el problema del marco (dificultad resolutiva) se estima sobre una misma interpretación del problema del marco (dificultad definicional), a saber, como un problema de relevancia. 
Damasio, justifica aún más nuestra elección. Al respecto, algunos investigadores como Megill y Cogburn (2005) utilizan, a modo de evidencia empírica del rol de las emociones a la hora de abordar el problema del marco, las historias clínicas de personas con centros emocionales dañados. El daño en algunas estructuras cerebrales afecta a algunos pacientes de manera tal que éstos resultan "carentes de emoción" exhibiendo déficits profundos en la toma de decisiones. Concretamente, los daños en algunas de estas estructuras cerebrales vinculadas a las emociones reflejan un comportamiento que se asemeja al de los robots de Dennett, ya mencionados, al no saber cómo resolver el problema del marco. En efecto, algunos de los pacientes que tienen daños cerebrales (especialmente en la corteza orbitofrontal y ventromedial) reflejan cierta vacilación entre dos extremos a la hora de determinar relevancia: o elijen de forma rápida pero incorrectamente o no son capaces de elegir en absoluto, paralizado por la consideración exhaustiva de los planes, opciones y consecuencias. El daño cerebral no permite superar el problema del marco: o se quedan paralizados examinando toda la información sin lograr determinar relevancia (como el último robot de Dennett que terminó explotando) o, si lo hacen, no se manifiestan adecuación ni con prontitud (como lo refleja el aspecto eficiente del problema del marco). El caso de un paciente llamado Elliot, quien antes de extirparle un tumor había sido un empleado y padre ejemplar, refleja estas afirmaciones. Luego de la operación de tumor, su personalidad cambió y comenzó a tener problemas para priorizar tareas; sufría el "problema del marco" pues sólo se centraba en hacer tareas triviales, o en aspectos irrelevantes de sus funciones laborales, sin atender a sus objetivos fundamentales, es decir, sin llevar a cabo adecuadamente la tarea de determinar relevancia entre mucha información. Parecería que en el caso de daño o de ausencia de contenido emocional, la cognición falla en el momento de determinar (adecuadamente) la relevancia entre vasta cantidad de información (Megill \& Cogburn, 2005, pp. 309-312). Siendo estas algunas de las razones que justifican nuestra elección comencemos pues por examinarla. La tesis principal de Damasio defiende primordialmente que las emociones representan estados corporales, o de otra manera, que las emociones son percepciones de un conjunto de respuestas neurofisiológicas coordinadas. Su teoría, denominada más específicamente como la teoría de los marcadores somáticos, afirma, entre otras cosas, que la emoción perceptiva informa e influye en la determinación de relevancia, al menos, para tareas consistentes en la toma de decisiones. Esta afirmación posibilita a algunos autores como Megill \& Cogburn (2005) a argumentar que la teoría de los marcadores somáticos de Damasio es de gran utilidad a la hora de resolver la dificultad de la vastedad de información del problema del marco, al menos en tareas consistentes 
en la toma de decisiones. Si bien no es explícitamente presentado por Damasio en términos de la dificultad de la vastedad de información, ante una tarea de toma de decisiones el agente se enfoca en las opciones que tiene y las consecuencias de tales opciones. Este es un gran recorte, pero todavía hay una gran cantidad de información por ponderar, lo cual, nuevamente, puede generar una parálisis en el agente. Pues bien, lo que la teoría de Damasio propone para superar esta dificultad es que se limita la cantidad de información como consecuencia del funcionamiento de los marcadores somáticos. Como los marcadores somáticos automáticamente detectan el valor (positivo/negativo) de las posibles consecuencias, éstos colaboran, expeditivamente, a "tamizar" la información sin que sea necesario hacer un examen exhaustivo de todas las opciones y consecuencias. A través de estos mecanismos se "traba" la conveniencia o no de algunas opciones y con ello, la motivación de perseguir o evitar, respectivamente, a esas opciones. La hipótesis del marcador somático "filtra" las opciones relevantes o desecha las irrelevantes con base a las sensaciones que provocan algunas opciones, lo que conducirá o a rechazar las opciones que no son favorables o a aceptar las favorables. Al resaltar algunas opciones que son peligrosas y eliminarlas rápidamente se restringe automáticamente las opciones a considerar y sus posibles consecuencias. La emoción, al actuar como guía conectiva, restringe de esta manera la cantidad de alternativas a considerar. El examen exhaustivo, ahora sobre una cantidad de información considerablemente menor, ya no sería tan arduo; al reducirse la cantidad de información, se aliviana la sobrecarga computacional y no hay dificultad, o problema del marco, por enfrentar.

Pues bien y como ya lo mencionamos, parecería que esclarecer a qué tipo de emociones se refiere Damasio en su propuesta resulta ser una tarea sencilla puesto que, como ya lo refleja la denominación "marcadores somáticos", se destaca primordialmente el rol de las emociones de tipo perceptivas. Su mecanismo implica la experiencia, la dimensión corporal, el cuerpo, o de otra manera, el sentir con el cuerpo. Nótese que en su propuesta se refleja claramente lo que hemos denominado el carácter experiencial/corporal de las emociones. La emoción para Damasio, notoriamente de tipo perceptiva, se centra en el cuerpo y remite a lo que el sujeto siente en esa y no otra situación determinada: el sujeto se encuentra "anclado" en esa situación particular. Claramente, las emociones a las que se refiere este autor son del tipo mencionado pues resultan como respuestas cuasi reflejas ante determinados estímulos, relacionadas con los cambios corporales que experimenta ese sujeto particular en esa situación particular. Sin embargo, creemos necesario realizar algunas aclaraciones que cuestionan la exclusividad 
del tipo de emociones perceptivas en la propuesta de Damasio. Para este autor, las emociones son percepciones de un conjunto de respuestas neurofisiológicas coordinadas, es decir son percepciones del cuerpo (Damasio, 2001, p. 140). Así, en esta definición se interpretan las emociones a través del modo fenoménico en el que son sentidas corporalmente, lo que, sin dudas, las clasifica como perceptivas. Pero, como puede observarse en la descripción de su mecanismo, el autor no asume que las emociones poseen un rol pasivo ni descarta su relación con otros estados cognitivos, lo que cuestiona su carácter exclusivamente perceptivo. En este sentido, en su descripción del mecanismo hace referencia a las emociones cognitivas en cuanto hace referencia a cierto $i$ ) carácter racional pero también, iii) un rol activo dentro del plano de la toma de decisiones puesto que su propuesta orienta a la acción. En otras palabras, en la propuesta de Damasio puede verse claramente el papel de las emociones de tipo perceptivas, aunque no exclusivamente, pues al destacar la naturaleza activa de las emociones dentro del proceso racional, el autor sugiere también el rol de las emociones de tipo cognitivas. En otros trabajos, y en aclaraciones anteriores, sin embargo, hemos sostenido que en su propuesta queda pendiente la explicación de la parte del mecanismo que lleva desde la percepción de la situación y entendimiento de la tarea a realizar, a enfocarse en opciones y consecuencias. Tampoco explica suficientemente el mecanismo por medio del cual el contenido corporal puede colaborar en la tarea de estimar cómo los seres humanos determinamos relevancia "adecuadamente". Así, aunque evidenciamos en la teoría de Damasio el reconocimiento del rol de las emociones cognitivas, consideramos que su propuesta destaca sobremanera el alcance de las emociones perceptivas, en tanto sensaciones fisiológicas. Por ello, la explicación de Damasio acerca de las emociones nos resulta incompleta e imparcial a la hora de resolver el problema del marco.

\section{Alcances y limitaciones del análisis}

Descritas distintas resoluciones, nuestro objetivo en esta sección es poner en tensión algunas de las características fundamentales de cada tipo de emoción a la hora de resolver el problema del marco. Nuestra postura, en acuerdo con algunos autores como Wild (2008) y Dohrn (2008) defiende que ni las emociones perceptivas ni las cognitivas son suficientes por sí solas para determinar relevancia, y con ello para resolver el problema del marco. Si bien nuestro análisis podría generalizarse al 
alcance de las emociones en general, para resolver el problema del marco preferimos aquí restringir nuestro análisis a las emociones cognitivas y a las perceptivas. Las investigaciones alrededor del rol de las emociones en nuestros procesos racionales aún se encuentran en estado incipiente, lo que posibilita que en un futuro no muy lejano se explicite con mayor precisión el rol de distintos tipos de emociones involucradas en procesos racionales. Pero además, aunque un tipo de emoción pueda contribuir directamente en la solución del problema del marco, no quita que otras lo hagan de manera indirecta. Esta postura, de tipo intermedia, es la que guiará a continuación nuestro análisis a propósito de los alcances y las limitaciones de cada tipo de emoción a la hora de resolver el problema del marco.

Comencemos con una de las críticas claves que algunos investigadores plantean frente a la propuesta de que sean las emociones perceptivas las que aborden teóricamente el problema del marco. El principal problema que este tipo de emoción afronta es explicar la manera en que las sensaciones pueden estar dirigidas hacia objetos o situaciones particulares del mundo; es decir, presentan algunos inconvenientes a la hora de explicar la naturaleza intencional de las emociones. ${ }^{3}$ Como consecuencia, y en este sentido, las emociones de tipo perceptivas resultan un tipo de fenómenos a los que no se les aplica las categorías de justificación, adecuación o racionalidad, categorías que sí podemos aplicar a las emociones de tipo cognitivas, al momento de estimar qué tan "adecuadamente" determinamos relevancia. Otra de las objeciones a las emociones de tipo perceptivas, en su versión más radical, es que heredan la antigua concepción de las emociones como estados pasivos, reduciéndolas a meras respuesta frente a determinados estímulos, concepción que actualmente está en discusión debido a la naturaleza activa de las emociones en nuestros procesos cognitivos. Continuando ahora con las objeciones a las emociones de tipo cognitivas, es interesante la que postula Griffiths (1997) al destacar que éstas descuidan el hecho de que no todo juicio de valor es emocional. La clase de los juicios de valor es mucho más amplia que la clase de los juicios de valor emocionales; que una persona estime que no comer sano es peligroso para la salud, no implica que coma una hamburguesa con miedo. Por lo tanto, una emoción de tipo cognitiva debería disipar juicios de valores emocionales y no emocionales en aquellos procesos cognitivos que implican relevancia. Otra de las críticas

3 Quizás convenga aclarar que la intencionalidad de los estados mentales a la que nos referimos en este trabajo está dirigida al mundo y no, tal como se refiere James (1989) hacia el propio organismo del sujeto y sus propios cambios fisiológicos. 
fundamentales a las emociones de tipo perpectivas es la dificultad a la hora de naturalizar los sentires corporales, es decir la dificultad para explicar los elementos corporales de la experiencia emocional al momento de determinar relevancia.

Al respecto, y reconociendo ahora los aportes de las emociones de tipo perceptivas, éstas destacan la ventaja de percibir cambios corporales, lo que permite dar una respuesta adaptativa frente a situaciones peligrosas como, por ejemplo, la huida. Así, las emociones de tipo perceptivas destacan las propiedades relacionales del agente con el entorno en el que se encuentra inmerso. Incluso para algunos autores este tipo de emociones se consideran "detectores de relevancia" (Prinz, 2004) cuya función es la de detectar ciertos rasgos del entorno que informan a los sujetos las propiedades relacionales con su entorno. Particularmente, ya hemos visto en la propuesta de Damasio, este carácter experiencial/corporal de las emociones perceptivas puesto que, de acuerdo a su propuesta, establecer relevancia implica la experiencia y la dimensión corporal, lo que da cuenta de la flexibilidad y adaptación con la que los seres humanos determinamos relevancia. Las emociones de tipo perceptivas se encienden, como "luces", ante la presencia de determinadas opciones o factores, estableciendo relevancia y orientándonos a la "acción". Pero no a cualesquiera de éstas, y teniendo en mente ahora el aspecto eficiente del problema del marco, sino de aquella acción que me lleve a actuar eficientemente, es decir adecuadamente y en un tiempo prudencial. Es aquí donde entra en juego: iii) las emociones de tipo cognitivas y su relación con el logro de objetivos frente a una situación determinada. Ya hemos destacado que el llevar a cabo una tarea eficientemente implica, entre otros varios factores, diversos objetivos en conflicto y un entorno muy variable. Teniendo en cuenta tales variables, las emociones de tipo cognitivas ofrecerían $i$ ) una valoración muy resumida de la situación que es importante para la acción. Considerar que ciertos aspectos de una situación resultan relevantes para lograr nuestra tarea, nos hace concentrarnos en ellas sin que nuestros limitados recursos cognitivos y el poco tiempo disponible se ocupe de cuestiones irrelevantes. Así las emociones de tipo cognitivas colaboran activamente en los procesos de relevancia a modo de "guía" entre vasta información, permitiendo así que, la mayoría de las veces, determinemos relevancia de manera eficiente.

Teniendo en cuenta los aportes de cada tipo de emoción para con la resolución del aspecto eficiente del problema del marco, asumimos que, en distinta medida, ambos tipos de emociones intervienen en su resolución. Más específicamente, el carácter experiencial/corporal propio de las emociones de tipo perceptivas y la 
relación que las emociones de tipo cognitivas establecen con el logro de objetivos frente a una situación determinada, colaboran en su resolución. Apreciando los aportes de las emociones de tipo cognitivas, consideramos que éstas contribuirán particularmente a que el agente, dentro de un tiempo prudencial y utilizando recursos cognitivos limitados, se concentre solamente en aquellos aspectos de una situación realmente importantes para actuar y lograr los objetivos preestablecidos sin perder tiempo en deducciones irrelevantes. En este sentido, y como lo propone De Sousa, las emociones de tipo cognitivas contribuirían a que el agente determine relevancia eficientemente sin quedar paralizado como lo ejemplifica el caso de los robots de Dennett. El sujeto, desde el enfoque de las emociones de tipo cognitivas, desempeñaría un papel activo en la generación de estados emocionales, resultando por lo tanto responsable, al llevar a cabo sus propios procesos de evaluación cognitiva, de la adecuación o inadecuación de sus reacciones emocionales ante ciertas situaciones. Recordemos que, en contraste con las emociones perceptivas, sí tiene sentido decir de las emociones cognitivas que son apropiadas o no, adecuadas o no, al momento de reaccionar de distintos modos ante determinadas situaciones. Así el rol de las emociones de tipo cognitivas al momento de determinar relevancia colaboraría directamente en la tarea de estimar cuán adecuadamente/eficientemente lo hacemos.

Apreciando ahora el alcance de las emociones de tipo perceptivas al momento de determinar relevancia, éstas nos permiten un tipo de experiencia exploratoria, cuyo objeto de exploración es el entorno en el que se encuentra inmerso el agente $\mathrm{y}$ en donde prevalece una relación práctica del agente con ese entorno. De otra manera, y frente a nuestra cuestión clave, proponemos un tipo de emoción con tintes relacionales en tanto que determinar relevancia adecuadamente y de acuerdo a los objetivos propuestos (emoción de tipo cognitiva) resulte como consecuencia de explorar experiencialmente/corporalmente el entorno que me rodea (emoción de tipo perceptiva). De otra manera, dar significancia, relevancia experiencialmente a determinados factores de una situación (emociones de tipo perceptivas), postula una relación práctica entre el agente y sus objetivos (emociones de tipo cognitivas). Recordemos que el problema del marco establece a un agente en relación práctica con el mundo, con un actuar, con un hacer, y esto de acuerdo a una tarea determinada. Uno de los elementos constituyentes de las emociones perceptivas es la relación práctica que el sujeto establece con el entorno en donde se encuentra inmerso. Sostenemos que intentar explicar cómo los seres humanos determinamos relevancia sin pensar en esa relación no tendría sentido. Pero además, cualquier solución que 
intente resolverlo, debe considerar también un tipo de actuar, frente a una tarea y con objetivos a cumplir, que permita estimar que lo hacemos adecuadamente.

Ahora bien, algunos autores cuestionan la coincidencia, o no, entre emociones de tipo cognitivas y perceptivas al momento de determinar relevancia sobre una misma situación. Para algunos autores, tal combinación resultaría un obstáculo, no permitiendo que lo hagamos eficientemente, mientras que, para otros, y tal como lo sostenemos nosotros, tal combinación facilita nuestros procesos cognitivos de relevancia. Veamos un ejemplo que lo ilustre.

Imaginemos el caso de un sujeto que tiene miedo a volar en avión por primera vez. Sostenemos que al estimar, evaluar, deliberar sobre distintas características previamente ignoradas y patrones previamente desconocidos al momento de subir a un avión, cada uno de los tipos de emociones examinados proporciona conocimientos distintos sobre un dominio particular sin resultar excluyentes en el proceso de determinación de relevancia, en este caso, frente a la decisión de subirse o no al avión. Más particularmente sostenemos que ambos tipos de emociones enriquecen el conocimiento acerca del contexto al que el sujeto se va a enfrentar, su primer vuelo en un avión. Cada tipo de emoción, perceptiva y cognitiva, resulta una fuente de saliencia, sensible o cognitiva que, en combinación, al momento de determinar relevancia, le permiten atender a semejanzas, diferencias, patrones y discrepancias de una misma situación que, de no combinarse, quizás pasaría por alto.

Puede el sujeto juzgar que volar en avión es seguro y aún sentir, a pesar de ello, pánico al subirse al avión. La reacción a no querer subirse a un avión, en la que se basan varias fobias, parece ser automática, y no depender del juicio que uno hace sobre determinada circunstancia. Pensar entrar en un avión es suficiente para que alguien que tiene miedo a volar en avión sienta pánico y decida, sobre esa información, no subirse. La información que le ofrece su propio cuerpo (sudoración, taquicardia, etc.) le da fuertes indicios acerca de que no le es conveniente subirse al avión. En este caso, es el propio cuerpo el que le ofrece información relevante al momento de tomar su decisión acerca de cómo viajar, lo que no lo convierte en una persona irracional puesto, y esta es nuestra postura, las sensaciones del propio cuerpo deben incluirse en el análisis del contenido de la experiencia del sujeto.

Volviendo a Elgin y De Sousa, para quienes las emociones son entendidas como juicos, el tipo de emociones que subyace a no querer subirse a un avión coinciden con las "emociones irracionales" ya que en su marco teórico, éstas son 
inexplicables. Volviendo a la coincidencia, o no, entre emociones cognitivas y perceptivas, estos autores sostienen que si las emociones perceptivas no coinciden con un juicio sobre ese mismo objeto, entonces esa emoción, y la decisión basada en ella, resulta irracional. ${ }^{4}$ Esta afirmación parece descansar sobre la asignación exclusiva del carácter de racional a las emociones de tipo cognitivas, relegando a las emociones de tipo perceptivas el carácter de irracional o "mal fundadas". En oposición a esta postura, sostenemos que en los procesos de relevancia aquí examinados ambos tipos de emociones son "bien fundadas" en el sentido que aportan información válida al momento de determinar relevancia.

De otra manera, al momento de asignar relevancia frente a una situación determinada, el agente percibe distintos y varios significados emocionales de su entorno, al que estima en su conjunto para hacerlo eficientemente. Puesto que los casos paradigmáticos de las emociones remiten al mundo, el carácter de intencionalidad hacia el mundo es un requisito necesario para poder estimar relevancia, pero también y puesto que no podemos aislarnos corporalmente del entorno en que nos encontramos inmersos, las emociones perceptivas que colaboran en la tarea de "anclarnos", "ajustarnos" a ese entorno, deben tener lugar. En síntesis, ambos tipos de emociones colaboran en la tarea de determinar relevancia, es decir, de resolver el problema del marco. Por supuesto, hay casos como la alegría de un lógico al demostrar un teorema, donde los objetos particulares de las emociones son proposiciones. Pero este caso no se encuentra entre los casos paradigmáticos que remiten al mundo, como tampoco lo son aquellos que se refieren a la imaginación o a la ficción del sujeto, como tenerles miedo a las brujas o a los extraterrestres.

Volviendo al caso del avión, le otorgamos vivencia al significado de las percepciones en el sentido en que nos proponemos atender al comportamiento del agente al momento de determinar relevancia en "ese" (y no otro) entorno. Teniendo en cuenta el modo en que este trabajo interpretamos el concepto de relevancia, entendemos que ésta enmarca una cuestión práctica entre el agente y el significado que emocionalmente le atribuimos a ese entorno, lo que configura nuestra experiencia emocional. Retomando el carácter experiencial/corporal de las emociones del tipo perceptivas: en el caso del avión es la percepción de mi cuerpo temblando y la de mi corazón latiendo apresuradamente, entre otros tantos síntomas, lo que me provoca pánico. La percepción de mi cuerpo forma parte

4 Es a propósito de esta postura que en la sección 3.1 aclaramos la exclusividad que sostiene De Sousa entre las emociones solamente de tipo cognitivas con el problema del marco. 
de la información que considero al decidir cómo viajar: si entro en pánico, esta percepción resulta fundamental al punto que es sobre ella que decido no viajar en avión aunque (y aquí la disidencia con las emociones de tipo cognitivas) esto no concuerde con aquella información que asegura muy poco riesgo de accidente. Si mi percepción del cuerpo me resulta "normal", es decir no percibo ningún cambio corporal, esta información no sopesará en absoluto. De todas maneras, en ambos casos, mi decisión será examinada teniendo en cuenta también los objetivos y la tarea encomendada (destacándose aquí el rol activo de la emociones de tipo cognitivas). Quizás mi decisión varíe de acuerdo al propósito de mi vuelo, de la urgencia que esto implique, de la posibilidad de considerar otros medios de transporte, entre otros. Incluso otros factores, como la de ir acompañados o no durante el viaje, forma parte de toda la información que poseo al momento de determinar relevancia. Esta situación ejemplifica la experiencia emocional del sujeto en donde sí se experimenta, "se siente" las vivencias implicadas en ciertos actos. Esta manera de entender la emoción implica la experiencia, en donde tanto la dimensión corporal, el cuerpo, o de otra manera, el sentir con el cuerpo (emociones perceptivas) como los juicios de valor sobre el mundo y otros estados cognitivos (emociones cognitivas) tienen el mismo valor a la hora de determinar relevancia.

\section{Comentarios finales}

Nuestro trabajo aborda uno de los debates más interesantes y actuales en la literatura del problema del marco: el rol de las emociones como vía promisoria para resolver el problema del marco. Claro está, y por cuestiones de espacio, profundizar tal debate nos hubiera llevado mucho más espacio del que disponemos, resultando necesario limitar nuestra tarea. Así, nos hemos restringido, con respecto al rol de las emociones, a una de sus principales clasificaciones, a saber aquella que distingue entre emociones de tipo cognitivas y de tipo perceptivas. Los aportes principales de este trabajo, teniendo en cuenta la poca investigación disponible, radicaron en i) sistematizar distintas propuestas de resolución del problema del marco y iii) atender a los alcances y limitaciones de cada tipo de emoción a la hora de resolver el aspecto eficiente del problema del marco. Argumentamos, asumiendo una posición intermedia, que ambos tipos de emociones poseen las herramientas necesarias a la hora de explicar cómo los seres humanos resolvemos el problema del marco debido, por un lado, al carácter experiencial/corporal propio de las emociones de tipo perceptivas y, por otro lado, a la relación que las emociones de tipo cognitivas 
establecen con el logro de objetivos frente a una situación determinada. Queda pendiente en nuestra agenda de investigación: $a$ ) qué sucede con respecto a otros aspectos (aspecto computacional, lógico, el epistemológico, el explicativo, etc.) del problema del marco, $b$ ) a qué contextos se aplica la propuesta que apela al rol de las emociones para resolverlo y, entre varias otras cuestiones, $c$ ) qué concepción de emoción contribuiría a su resolución. Asimismo, el restringir nuestro análisis a solamente dos tipos de emociones, y no a todas y a cada una de ellas, convierte a este trabajo en parte de un proyecto más general, que sin dudas, continuaremos desarrollando en próximos trabajos de investigación.

\section{Referencias}

Brun, G., Ulvi D. \& Dominique K. (2008). Epistemology and emotions. Aldershot: Ashgate.

Crockett, L. (1994). The Turing Test and the Frame Problem: AI's mistaken understanding of intelligence. New Yersey: Ablex Publishing Corporation.

Damasio, A. (2001). El error de Descartes: la emoción, la razón y el cerebro humano. Barcelona: Crítica.

Damasio, A. (2010). Self comes to mind. New York: Pantheon.

De Sousa, R. (1980). The rationality of emotion. En R. Amelie (Ed.), Explaining emotions (pp. 127-151). Berkeley: University of California Press.

De Sousa, R. (1996). Emotion. En S. Guttenpland (Ed.), A companion to the philosophy of mind (pp. 270-276). Oxford: Basic Blackwell.

Dennett, D. (1984). Cognitive wheels: The frame problem of AI. En C. Hookway (Ed.), Minds, machines \& evolution (pp. 129-150). Cambridge: Cambridge University Press.

Dohrn, D. (2008). Epistemic immediacy and reflection. En G. Brun, U. Doguoglu \& D. Kuenzle (Eds.), Epistemology and emotions (pp. 105-124). Aldershot: Ashgate.

Elgin, C. (1996). Considered judgment. Princeton: Princeton University Press.

Elgin, C. (2008). Emotion and Understanding. En G. Brun, U. Doguoglu \& D. Kuenzle, Epistemology and emotions (pp. 33-49). Alderchot: Ashgate.

Ekman, P. (1993). Facial expressions and emotion. American Psychological Association, 48, 376-379. https://doi.org/10.1002/0470013494.ch16

Ekman, P. \& Friesen W. V. (1971). Constants across cultures in the face and emotion. Journal of Personality and Social Psychology, 17, 124-129. http://dx.doi.org/10.1037/h0030377

Ekman P., Sorenson, E. R., \& Friesen, W. (1969). Pan-cultural elements in facial displays of emotion. Science, 164, 86-88. http://dx.doi.org/10.1126/science.164.3875.86

Griffiths, P. (1997). What emotions really are: the problem of psychological categories. Chicago: The University of Chicago Press.

Hansberg, O. (1996). La diversidad de las emociones. México: Fondo de Cultura Económica. 
Hjørland, B. (2010). The foundation of the concept of Relevance. Journal of the American Society for Information Science and Technology, 61(2), 217-237. https://doi.org/10.1002/asi.21279.

James, W. (1985). ¿Qué es una emoción? Estudios de Psicología, 21, 57-73. https://doi.org/10 $.1080 / 02109395.1985 .10821418$

James, W. (1989). Principios de Psicología. México: Fondo de Cultura Económica.

Kamermans M. \& Schmits T. (2004). The History of the Frame Problem. Amsterdam: UniversityFaculty of Artificial Intelligence. https://doi.org/10.1016/B978-0-934613-32-3.50004-6

Lange, W. (1885). The mechanism of emotions. En R. Brand (Ed.), The classical psychologists: selections illustrating psychology from Anaxagoras to Wundt (pp. 672-684). Boston: Houghton Mifflin.

Lowe, E. (2001). Filosofia de la mente. Barcelona: Idea Universitaria.

McCarthy, J. \& Hayes, P. (1969). Some philosophical problems from the standpoint of AI. Machine Intelligence, 4, 463-502. https://doi.org/10.1016/B978-0-934613-03-3.50033-7

Megill, J. \& Cogburn, J. (2005). Easy's getting harder all the time: Human emotions and the Frame Problem. Ratio, 18(3), 306-316. https://doi.org/10.1111/j.1467-9329.2005.00292

Nussbaum, M. (2008). Paisajes del pensamiento. Barcelona: Paidós.

Oatley, K. (1992). Best laid schemes: The psychology of emotions. Cambridge: Cambridge University Press.

Pinker, S. (2005). So how does the mind work? Mind \& Language, 20(1), 1-24. https://doi.org /10.1111/j.0268-1064.2005.00274.

Prinz, J. (2004). Gut reactions: A perceptual theory of emotion. New York: Oxford University Press.

Prinz, J. (2006). Is an emotion a form of perceptions? Canadian Journal of Philosophy, 36, 137-160. https://doi.org/10.1353/cjp. 2007.0035.

Plutchik, R. (1980). Emotion: a psychoevolutionary synthesis. New York: Harper \& Row. https:// doi.org/10.1016/B978-0-12-558701-3.50007-7.

Schneider, S. (2007). Yes, it does: a diatribe on Jerry Fodor's "The mind doesn't work, that way". Psyche, 13(1), 1-15. https://doi.org/10.1080/0951508021000042067

Schwitzgebel, E. (2010). Acting contrary to our professed beliefs or the gulf between occurrent judgment and dispositional belief. Pacific philosophical Quarterly, 91(4) 531-553. https:// doi.org/10.1111/j.1468-0114.2010.01381.

Shanahan, M. (1997). Solving the frame problem: A mathematical investigation of the commonsense law of inertia. Cambridge: The Mit Press.

Silenzi, M. I. (2014). El problema de marco: alcances y limitaciones de los enfoques Postcognitivistas. Bahía Blanca: Ediuns.

Silenzi, M. I. (2015a). ¿En qué consiste el problema de marco? Confluencias entre distintas interpretaciones, Eidos: Revista de Filosofía de la Universidad del Norte, 22, 49-80. http:// dx.doi.org/10.14482/eidos.22.6381.

Silenzi M. I. (2015b). La dualidad del Problema de marco: Sobre interpretaciones y resoluciones, 
El rol de las emociones a la hora de resolver el problema del marco...

Revista Tópicos: Revista de Filosofía, 47, 89-112. https://doi. org/10.21555/top. v0i47.663. Silenzi M. I. (2015c). Enfoques postcognitivistas: rótulos, presupuestos y posibles lecturas, Ludus Vitalis: Revista de filosofia de las ciencias de la vida. 23(43) 277-288.

Sperber D. y Deirdre W. (1986). Relevance: Communication and Cognition. Cambridge: Harvard University Press.

Solomon, R. (1993). The passions: Emotions and the meaning of life. Indianapolis: Hackett Publishing Company.

Tappolet, C. (2000). Émotions et Valeurs. Paris: Presses Universitaires de France.

Thagard, P. (2008). La mente: introducción a las ciencias cognitivas. Buenos Aires: Katz.

Wild, M. (2008). Critical reflection on affective epistemology. En G. Brun, U. Doguoglu \& D.

Kuenzle. Epistemology and emotions (pp. 125-148). Alderchot: Ashgate. 